\title{
Terahertz Light-Matter Interaction beyond Unity Coupling Strength
}

\author{
Andreas Bayer, Marcel Pozimski, Simon Schambeck, Dieter Schuh, Rupert Huber, Dominique Bougeard, \\ and Christoph Lange*(0)
}

Department of Physics, University of Regensburg, 93040 Regensburg, Germany

\section{Supporting Information}

ABSTRACT: Achieving control over light-matter interaction in custom-tailored nanostructures is at the core of modern quantum electrodynamics. In strongly and ultrastrongly coupled systems, the excitation is repeatedly exchanged between a resonator and an electronic transition at a rate known as the vacuum Rabi frequency $\Omega_{R}$. For $\Omega_{R}$ approaching the resonance frequency $\omega_{c}$, novel quantum phenomena including squeezed states, Dicke superradiant phase transitions, the collapse of the Purcell effect, and a population of the ground state with virtual photon pairs are predicted. Yet, the experimental realization of optical systems with $\Omega_{\mathrm{R}} / \omega_{\mathrm{c}} \geq 1$ has remained elusive. Here, we introduce a paradigm
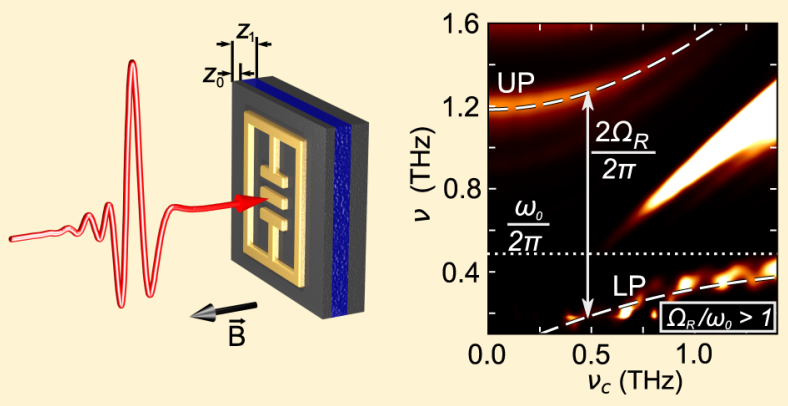

change in the design of light-matter coupling by treating the electronic and the photonic components of the system as an entity instead of optimizing them separately. Using the electronic excitation to not only boost the electronic polarization but furthermore tailor the shape of the vacuum mode, we push $\Omega_{R} / \omega_{c}$ of cyclotron resonances ultrastrongly coupled to metamaterials far beyond unity. As one prominent illustration of the unfolding possibilities, we calculate a ground state population of 0.37 virtual photons for our best structure with $\Omega_{R} / \omega_{c}=1.43$ and suggest a realistic experimental scenario for measuring vacuum radiation by cutting-edge terahertz quantum detection.

KEYWORDS: Quantum electrodynamics, ultrastrong coupling, terahertz, metamaterials

$I^{n}$ $\mathrm{n}$ the strong coupling regime of quantum electrodynamics (QED), where the vacuum Rabi frequency $\Omega_{\mathrm{R}}$ exceeds the dissipation rates of the electronic excitation and the resonator, new eigenmodes called cavity polaritons emerge. This universal principle is found in a large variety of systems, ranging from atoms ${ }^{1}$ to excitons in semiconductors, ${ }^{2,3}$ molecules, ${ }^{4}$ mid-IR plasmonic structures, ${ }^{5-9}$ circuit $\mathrm{QED}$ systems at $\mathrm{GHz}$ frequencies, ${ }^{10-13}$ and structures in the $\mathrm{THz}$ spectral range. ${ }^{14-16}$

In ultrastrongly coupled structures, $\Omega_{\mathrm{R}}$ becomes comparable to the resonance frequency $\omega_{\mathrm{c}}$ itself; the rotating-wave approximation of light-matter interaction falters, and antiresonant coupling terms describing the simultaneous creation of correlated light and matter excitations become relevant. ${ }^{17-19}$ Most prominently, the ground state is theorized to be a modified squeezed quantum vacuum with a finite population of correlated virtual photon pairs. ${ }^{17,19}$ For sufficiently large values of the relative coupling strength $\Omega_{\mathrm{R}} / \omega_{\mathrm{c}} \gtrsim 1$, subcycle switching of $\Omega_{R}{ }^{6,9}$ may release these photons ${ }^{17,19,20}$ in analogy to UnruhHawking radiation emerging at the event horizon of black holes. ${ }^{21}$ These spectacular perspectives have fuelled the quest of the QED community for ever greater relative coupling strengths, ultimately aiming for $\Omega_{\mathrm{R}} / \omega_{\mathrm{c}}$ beyond unity.

The key strategy for boosting $\Omega_{\mathrm{R}} / \omega_{\mathcal{\mathcal { O }}}$ also referred to as $g / \omega_{\mathcal{c}}$ comprises increasing the dipole moment of the electronic transition, decreasing the resonator mode volume and $\omega_{\mathfrak{c}}$, or enhancing the overlap of the photonic mode and the electronic polarization field. Following these considerations, the regime of ultrastrong coupling was first established using intersubband transitions of quantum wells (QWs) coupled to mid-infrared or $\mathrm{THz}$ waveguides ${ }^{5-7,9}$ or plasmonic dot cavities, ${ }^{8,14}$ achieving values on the order of $\Omega_{\mathrm{R}} / \omega_{\mathrm{c}} \approx 0.25 .{ }^{14}$ Circuit $\mathrm{QED}$ systems, where excitations of superconducting resonators are coupled to superconducting two-level systems at $\mathrm{GHz}$ frequencies, followed up shortly ${ }^{10}$ and recently enabled above-unity coupling strengths of up to $\Omega_{\mathrm{R}} / \omega_{\mathrm{c}}=1.34 .{ }^{12,13}$ In comparison, in the domain of radiative coupling, the giant dipole moment of cyclotron resonances $(\mathrm{CR})$ coupled to planar metamaterials has enabled a milestone value of $\Omega_{R} / \omega_{c}=0.87,{ }^{15,16}$ highlighting key advantages of the CR for $\mathrm{QED}^{22,23}$ such as the tunability by the magnetic bias or control of the oscillator strength by the Landau level filling factor.

Here, we report an unprecedentedly large relative coupling strength of $\Omega_{\mathrm{R}} / \omega_{\mathrm{c}}=1.43$ in planar $\mathrm{THz}$ metamaterials ultrastrongly coupled to CRs. In particular, we tailor the vacuum mode of the structure by both, the metamaterial and the electronic excitation. In conventional design approaches, where the electronic and photonic components are typically treated separately, the electronic resonance is placed in a maximum of the light field calculated for the empty resonator. For $\Omega_{\mathrm{R}} / \omega_{\mathrm{c}}$ exceeding unity, however, the electronic resonance has been predicted to strongly influence the light field, ${ }^{18}$

Received: July 20, 2017

Revised: $\quad$ August 31, 2017

Published: September 22, 2017 


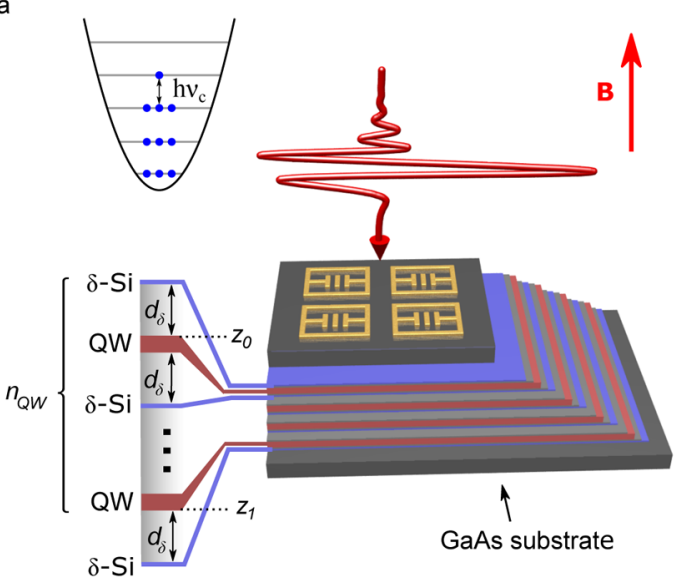

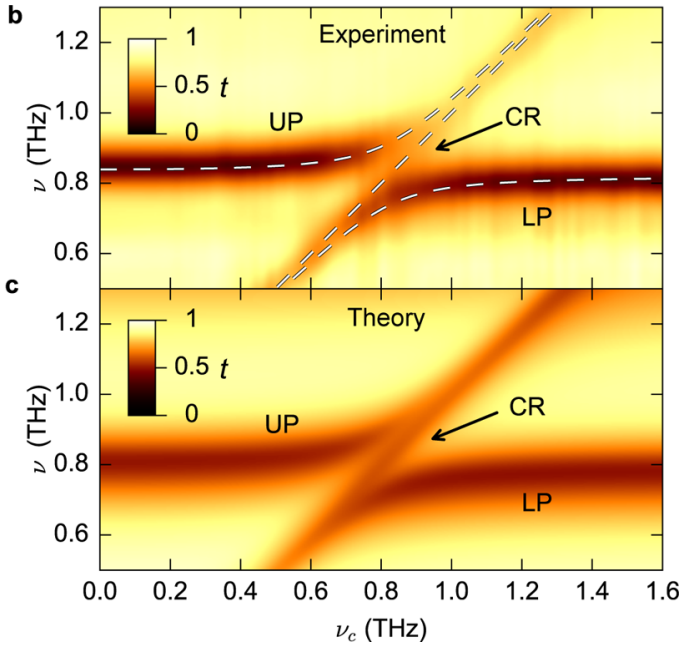

Figure 1. Ultrastrong coupling of $\mathrm{THz}$ metamaterials to the cyclotron resonance of 2DEGs. (a) Experimental geometry of magneto-THz transmission measurements. Red arrow: $B$-field. QW: quantum wells. $\delta$-Si: doping layers. $z_{0}, z_{1}$ : Upper and lower extension limit of the QW stack, respectively. Inset: Landau level fan of magnetically biased two-dimensional electron gas. (b) Color plot of measured transmission as a function of $\nu_{\mathrm{c}}$. Dashed curves: Dispersion of the lower (LP) and upper polariton (UP) determined by fitting the transmission minima to the Hopfield Hamiltonian, which yields $\Omega_{\mathrm{R}} / \omega_{\mathrm{c}}=0.11$. Diagonal line: cyclotron resonance. (c) Calculated transmission spectrum, and resonances labeled as in panel b. The Hopfield fit yields $\Omega_{\mathrm{R}} / \omega_{\mathrm{c}}=0.10$.

Table 1. Structural and Optical Parameters of All Samples

\begin{tabular}{|c|c|c|c|c|c|c|c|}
\hline structure & $n_{\mathrm{QW}}$ & $\rho\left(10^{12} \mathrm{~cm}^{-2}\right)$ & $z_{0}(\mathrm{~nm})$ & $z_{1}(\mathrm{~nm})$ & $d_{\delta}(\mathrm{nm})$ & $\Omega_{\mathrm{R}} / \omega_{\mathrm{c}}$ (exp.) & $\Omega_{\mathrm{R}} / \omega_{\mathrm{c}}$ (theory) \\
\hline 1 & 1 & 0.30 & 500 & & 30 & 0.11 & 0.10 \\
\hline 2 & 25 & 0.54 & 80 & 3105 & 50 & 0.53 & 0.57 \\
\hline 3 & 6 & 1.85 & 325 & 535 & 15 & 0.62 & 0.66 \\
\hline 4 & 6 & 1.38 & 50 & 310 & 20 & 0.99 & 1.09 \\
\hline 5 & 6 & 1.75 & 45 & 255 & 15 & 1.13 & 1.21 \\
\hline 6 & 6 & 3.00 & 40 & 200 & 10 & 1.43 & 1.57 \\
\hline
\end{tabular}

requiring a joint treatment of both components. We have thus developed a parameter-free model of light-matter coupling which renders the electric field distribution on extremely subwavelength scales, enables predictive design of nanoscale QED structures, and an unambiguous identification of polariton modes. Exceeding unity relative coupling strength in the optical regime, for the first time, our structures enter uncharted terrain of nonperturbative light-matter dynamics, bringing theoretically discussed quantum phenomena expected for $\Omega_{\mathrm{R}} / \omega_{\mathrm{c}}>1$ within experimental reach. ${ }^{17-19,24,25}$ In particular, we give a quantitative experimental scenario for the most spectacular prediction, the detection of optical quantum vacuum radiation.

We employ high-quality quantum wells hosting two-dimensional electron gases which are Landau-quantized by an external magnetic field of up to $B=5 \mathrm{~T}$. The emerging CR exhibits a frequency of $v_{\mathrm{c}}=e B / 2 \pi m^{*}$ freely tunable by the $B$-field, where $e$ is the elementary charge and $m^{*}$ is the electron effective mass. Arrays of $\mathrm{THz}$ resonators processed on top of the QW stack comprise the photonic parts of our structures, where each resonator independently provides a localized confined optical mode which couples to the $\mathrm{CR}$, forming confined cavity polaritons. The transmission spectrum is measured using $\mathrm{THz}$ time-domain magneto-spectroscopy (Figure 1a). Structural and optical parameters are summarized in Table 1.

We first investigate a conceptually straightforward structure consisting of a single QW with a doping density of $\rho=3.0 \times$ $10^{11} \mathrm{~cm}^{-2}$, located at a depth $z_{0}=500 \mathrm{~nm}$ below the surface, and a double-gap metamaterial with a resonance frequency of $v_{\mathrm{m}}=0.82 \mathrm{THz}$ (Figure 1a and Supporting Information). The transmission spectra as a function of $v_{c}$ show the lower polariton (LP) which merges into the metamaterial resonance for large $v_{\mathcal{C}}$ and the upper polariton (UP) (Figure 1b, dashed lines). On resonance, $v_{\mathrm{c}}=v_{\mathrm{m}}$, the typical anticrossing signature is observed and the spectral separation of LP and UP with frequencies of $\nu_{\mathrm{LP}}=0.74 \mathrm{THz}$ and $\nu_{\mathrm{UP}}=0.92 \mathrm{THz}$, respectively, is minimal. In addition, the bare $\mathrm{CR}$ originating from uncoupled areas of the structure is visible as a diagonal line. Using a fitting procedure based on the Hopfield formalism which takes the entire polariton frequency response into account, ${ }^{15}$ we determine a coupling strength of $\Omega_{\mathrm{R}} / \omega_{\mathrm{c}}=0.11$.

We numerically calculate the complex field distribution and transmission spectra by a classical electrodynamical theory. Our formalism models the dielectric environment of the nanostructure and implements the $\mathrm{CR}$ as a gyrotropic medium, allowing for a unified treatment of photonic and electronic components and accounting for their mutual interplay (see Supporting Information). The resulting far-field calculation (Figure 1c) predicts the experimental transmission across the entire spectral range with high accuracy, and the Hopfield fit yields $\Omega_{\mathrm{R}} / \omega_{\mathrm{c}}=$ 0.10 .

As a first measure to increase $\Omega_{R} / \omega_{c}$, we exploit the relation $\Omega_{\mathrm{R}} / \omega_{\mathrm{c}} \propto\left(n_{\mathrm{OW}} \times \rho\right)^{1 / 2}($ ref 26$)$ and boost the macroscopic electronic polarization of the ensemble of Landau-quantized electrons by increasing $\rho$ to $5.4 \times 10^{11} \mathrm{~cm}^{-2}$. We furthermore employ $n_{\mathrm{OW}}=25$ QWs of a thickness $d_{\mathrm{OW}}=25 \mathrm{~nm}$, each, separated from their doping layers by $d_{\delta}=50 \mathrm{~nm}$ to form a stack extending from $z_{0}=80 \mathrm{~nm}$ to $z_{1}=3.1 \mu \mathrm{m}$ (cf. Figure $1 \mathrm{a}$ ). These modifications are accompanied by significantly more 

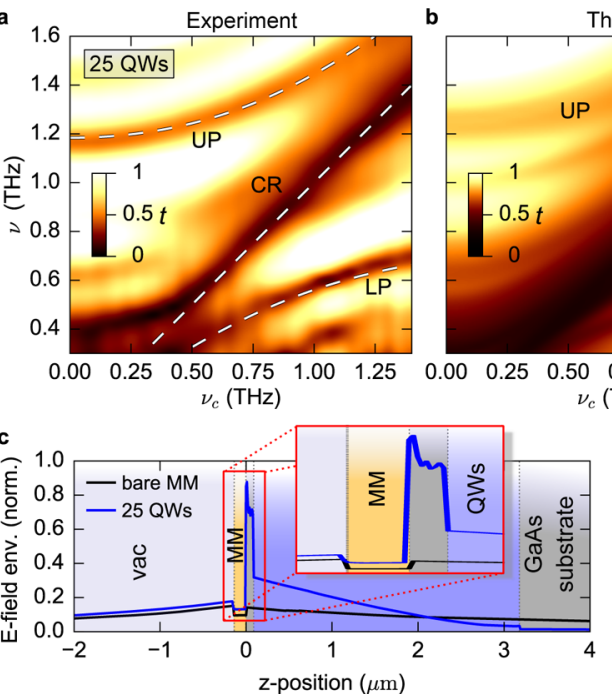
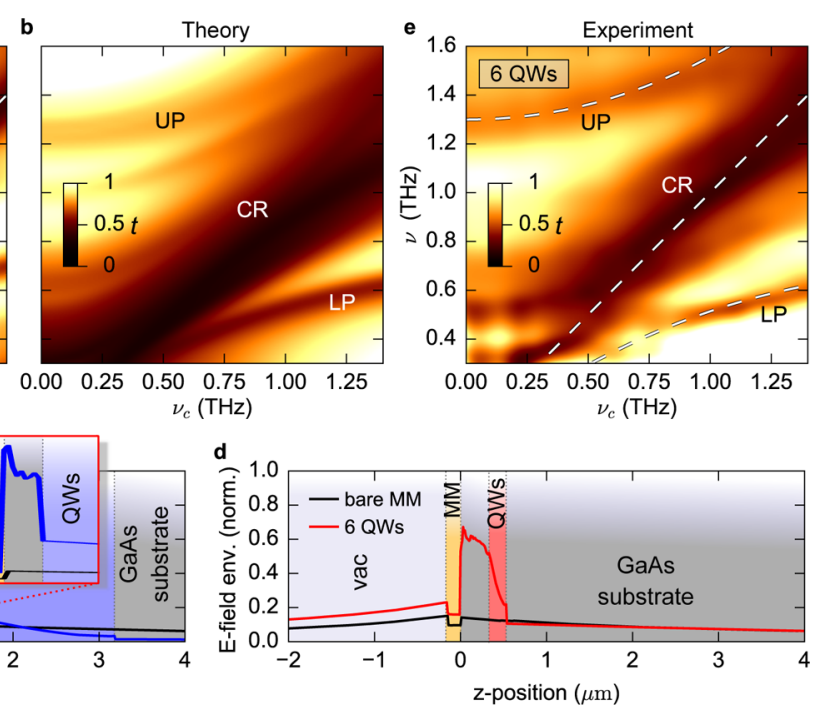

Figure 2. Shaping of the vacuum mode by tailoring of the electronic excitation. (a) Color plot of the experimental transmission spectra of the 25-fold QW stack (structure 2). Dashed curves: polariton resonances obtained by Hopfield fit, which yields $\Omega_{\mathrm{R}} / \omega_{\mathrm{c}}=0.53$. (b) Numerically calculated transmission (Hopfield fit: $\Omega_{\mathrm{R}} / \omega_{\mathrm{c}}=0.57$ ). (c) Electric field envelope of the LP mode of structure 2, in $z$-direction (blue curve), averaged across the $x-y$-plane. The QW stack is shown as a blue-shaded area. The magnified area highlights the mode localization between the metamaterial and the QWs. Black curve: Envelope of the bare metamaterial. (d) Significantly enhanced mode confinement of the 6-fold QW stack of structure 3 (red curve) and (e) resulting experimental transmission spectra, for which the Hopfield fit yields $\Omega_{\mathrm{R}} / \omega_{\mathrm{c}}=0.62$.

complex transmission spectra (Figure 2a) which exhibit multiple resonances, requiring a strategy to unambiguously identify the polariton modes. Our theory matches the experimental data accurately (Figure 2b), enabling this identification by two independent methods: First, we calculate the frequency evolution of each mode as a function of $\rho$ and verify that LP and UP merge into the metamaterial resonance for $\rho \rightarrow 0$, without any discontinuities. Second, we compare the spatial shapes of the polariton and metamaterial modes. Following these procedures (see Supporting Information), we pinpoint the LP and UP (dashed curves) with center frequencies of $\nu_{\mathrm{LP}}=0.53 \mathrm{THz}$ and $\nu_{\mathrm{UP}}=1.38 \mathrm{THz}$ at the anticrossing point and $\Omega_{\mathrm{R}} / \omega_{\mathrm{c}}=0.53$ as confirmed by our calculation, which yields $\Omega_{\mathrm{R}} / \omega_{\mathrm{c}}=0.57$.

Analyzing the full spatial electric field distribution of the coupled system demonstrates that a separate treatment of the resonator and the electronic transition cannot accurately describe the present scenario: The envelope function of the LP mode in $z$-direction (Figure 2c, blue curve) significantly deviates from the mode of the bare resonator (black curve). In particular, the dense carrier plasma of the QWs (blue-shaded area) acts similarly to a mirror-a phenomenon that has been predicted to cause light-matter decoupling in a mirror-defined microcavity. ${ }^{18}$ In the near-field configuration present in our structures, in contrast, the interplay of the electronic transition with the resonator mode can be exploited to further reduce the mode volume on a drastic subwavelength scale. By choosing a carrier density $\rho$ that leads to a strong expulsion of the electric field out of the QW stack, we considerably compress the optical mode by localizing it between the QW stack and the metamaterial (Figure 2c, inset). Despite the high doping level, the locally enhanced electric field still penetrates the QWs sufficiently to maintain a high spatial overlap of the confined mode with the electronic polarization, leading to an overall increase of $\Omega_{\mathrm{R}} / \omega_{\mathrm{c}}$. The depth of the high-field region is set by the structural parameter $z_{0}$, whereas $d_{\delta}$ and $\rho$ control the mode's exponential decay inside the stack.
The effectiveness of this approach is confirmed numerically in the more compact QW stack of structure 3 with the same metamaterial, where the localization of the mode is drastically enhanced, leading to an increased field amplitude inside the QW stack (Figure $2 \mathrm{~d}$, red curve). Structure 3 is realized by reducing $d_{\delta}$ to $15 \mathrm{~nm}$ and $d_{\mathrm{QW}}$ to $10 \mathrm{~nm}$ in a 6-fold QW stack, corresponding to a 14-fold reduction of the QW stack extension (Table 1 ), while $n_{\mathrm{QW}} \times \rho$ is reduced by just $20 \%$ compared to structure 2 (see Supporting Information). The spectra (Figure 2e) remain qualitatively similar to structure 2 for both the experiment and the simulation. However, we determine $\nu_{\mathrm{LP}}=0.45 \mathrm{THz}$ and $\nu_{\mathrm{UP}}=1.47 \mathrm{THz}$ at the anticrossing point, and the Hopfield fitting procedure yields an increased $\Omega_{\mathrm{R}} / \omega_{\mathrm{c}}=0.62$ in very good agreement with our numerical calculation $\left(\Omega_{\mathrm{R}} / \omega_{\mathrm{c}}=0.66\right)$.

Following this promising route of shaping the vacuum mode by the $2 \mathrm{DEG}$, we push the limits of expitaxial heterostructure growth in a series of three 6-fold QW structures. We improve the mirror-like role of the $2 \mathrm{DEG}$ by simultaneously optimizing $d_{\delta}$ for more compact QW stacks and $\mathrm{z}_{0}$ for higher mode localization. Exploiting the scaling $\Omega_{\mathrm{R}} / \omega_{\mathrm{c}} \propto \omega_{\mathrm{c}}^{-1 / 2}$ (ref 26), we employ a metamaterial with $v_{\mathrm{m}}=0.48 \mathrm{THz}$ implemented as an inverted structure following Babinet's principle, ${ }^{27}$ such that LP and UP manifest themselves as local transmission maxima.

For structure 4, the LP and UP resonances emerge as distinct features (Figure 3a) separated by $0.95 \mathrm{THz}$ at the anticrossing point, corresponding to $\Omega_{\mathrm{R}} / \omega_{\mathrm{c}}=0.99$ (theory: $\Omega_{\mathrm{R}} / \omega_{\mathrm{c}}=1.09$, Figure $3 \mathrm{~b})$. Intriguingly, light-matter coupling in these structures is strong enough to push a second-order lower polariton branch labeled LP2, resulting from strongly offresonant coupling of the $\mathrm{CR}$ to a higher mode of the metamaterial (see Supporting Information), into the spectral range of the fundamental LP and UP. Structures 5 and 6 exhibit qualitatively identical spectra (Figures $3 c, d$ ) well-reproduced by our theory (Figure $3 \mathrm{e}$ ). Yet, the LP and UP resonances are pushed even further apart (Figure 3f, top) resulting in coupling strengths of $\Omega_{\mathrm{R}} / \omega_{\mathrm{c}}=1.13$ (theory: $\Omega_{\mathrm{R}} / \omega_{\mathrm{c}}=1.21$ ) and $\Omega_{\mathrm{R}} / \omega_{\mathrm{c}}$ 

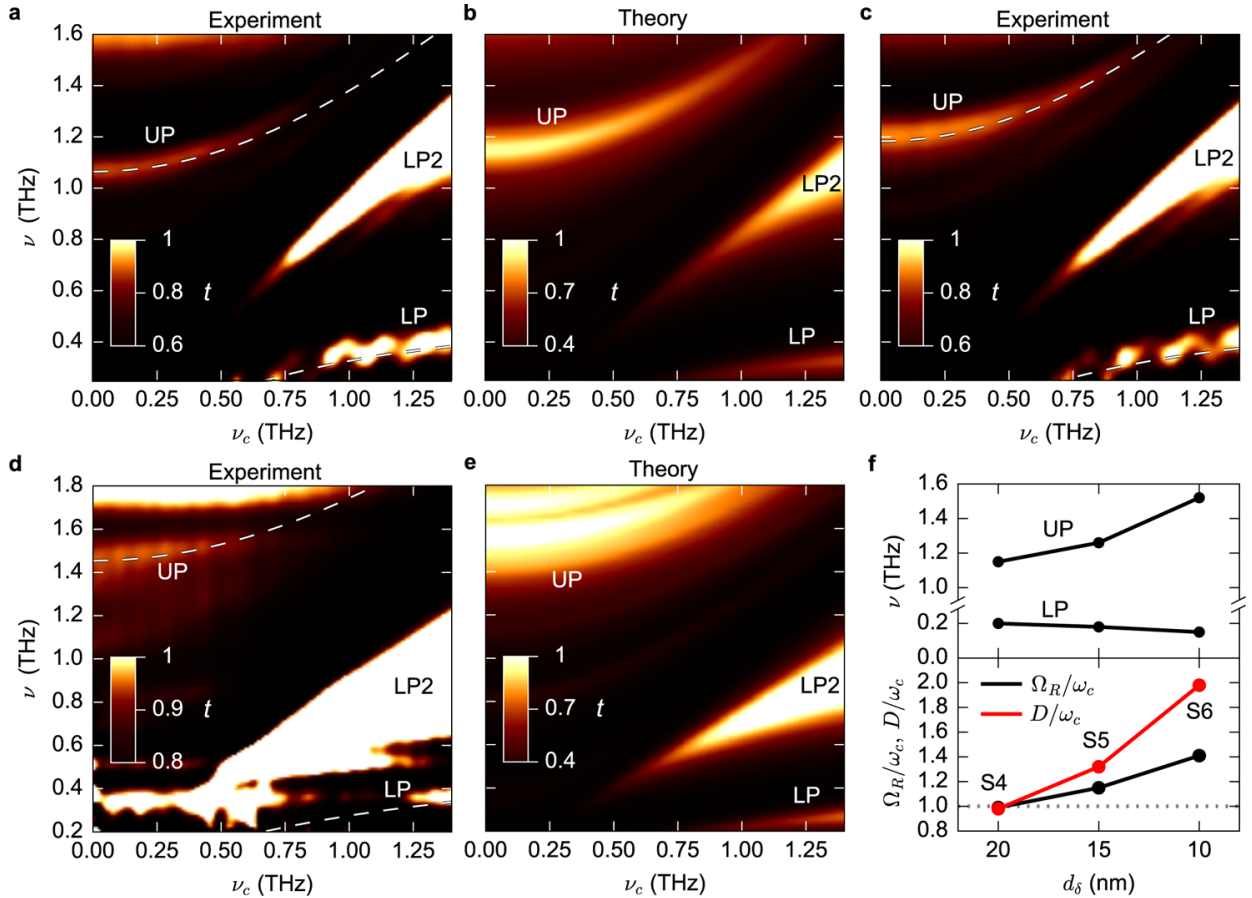

Figure 3. Coupling of inverted metamaterials and CR of extremely compact 6-fold QW stacks. (a) Transmission color plot of structure 4 with $d_{\delta}=$ $20 \mathrm{~nm}$ and $\rho=1.38 \times 10^{12} \mathrm{~cm}^{-2}$. LP and UP are evident as local transmission maxima and $\Omega_{\mathrm{R}} / \omega_{\mathrm{c}}=0.99$. LP2: higher-order coupled mode (see text). (b) Corresponding calculation. (c) Structure 5; $d_{\delta}=15 \mathrm{~nm}, \rho=1.75 \times 10^{12} \mathrm{~cm}^{-2}, \Omega_{\mathrm{R}} / \omega_{\mathrm{c}}=1.13$. (d) Structure $6 ; d_{\delta}=10 \mathrm{~nm}, \rho=3.00 \times 10^{12}$ $\mathrm{cm}^{-2}, \Omega_{\mathrm{R}} / \omega_{\mathrm{c}}=1.43$, and (e) calculation. (f) $v_{\mathrm{LP}}$ and $v_{\mathrm{UP}}$ for the 6-fold QW structures 4-6 (S4-S6) (top) and Rabi $\left(\Omega_{\mathrm{R}}\right)$ and diamagnetic coupling frequency $(D)$ at the anticrossing point (bottom).

$=1.43$ (theory: $\Omega_{R} / \omega_{c}=1.57$ ), respectively. These values mark the first realization of light-matter coupling strengths exceeding unity, in an optical system. Our optimization process demonstrates the power of the classical subwavelength calculations for boosting $\Omega_{R} / \omega_{c}$ by precisely predicting transmission spectra and revealing the role of tuning parameters such as $\rho, z_{0}$, and $d_{\delta}$ (Supporting Information), while allowing for unambiguous identification of spectral features.

At a relative coupling strength of $\Omega_{R} / \omega_{c}=1.43$ we enter a completely new regime for optical systems where light-matter energy exchange occurs on a time scale significantly shorter than the lifetime of vacuum fluctuations as permitted by the energy-time uncertainty principle, boosting the virtual photon population of the ground state $|G\rangle .{ }^{17}$ Here, a formal treatment of the vacuum requires a full quantum theory, including antiresonant light-matter interaction terms, $H_{\text {anti }}=$ $\mathrm{i} \hbar \Omega_{R, k}\left(\hat{a}_{k} \hat{b}_{-k}-\hat{a}_{k}^{\dagger} \hat{b}_{-k}^{\dagger}\right)+\hbar D_{k}\left(\hat{a}_{k} \hat{a}_{-k}-\hat{a}_{k}^{\dagger} \hat{a}_{-k}^{\dagger}\right)$, where $\hat{a}_{k}$ and $\hat{b}_{k}$ represent the annihilation operators for photon and matter excitations in mode $k$, respectively. ${ }^{17}$ The latter term is linked to the virtual photon population of the ground state ${ }^{17,19}$ and is proportional to the diamagnetic interaction strength $D_{k} \approx \Omega_{R, k}^{2}$ / $\omega_{c}$ which scales quadratically with $\Omega_{R}$, on resonance. For structure $6, D_{k} / 2 \pi=0.98 \mathrm{THz}$ even exceeds the vacuum Rabi frequency $\Omega_{R} / 2 \pi=0.69 \mathrm{THz}$; that is, antiresonant exchange dominates over vacuum Rabi interactions (Figure $3 \mathrm{f}$ ), and a vacuum photon population of $\left\langle G\left|\hat{a}_{k}^{\dagger} \hat{a}_{k}\right| G\right\rangle=0.37$ results. This novel scenario now enables experiments where diabatic switching ${ }^{6,9}$ of $10^{3}$ resonators should lead to $\mathrm{THz}$ field amplitudes of $0.15 \mathrm{~V} / \mathrm{cm}$, at the detector (Supporting Information). The incoherent nature of quantum vacuum radiation necessitates quantum detection ${ }^{28,29}$ and statistical analysis, for which we estimate that extremely low-noise electro-optic detection ${ }^{30,31}$ enables single-shot sensitivities of $0.8 \mathrm{~V} / \mathrm{cm}$ in a realistic experiment. Applying this detection scheme to our structures should enable the clear isolation of quantum vacuum radiation from the noise floor within a few seconds of acquisition time (Supporting Information).

Our approach introduces a paradigm change for the design of ultrastrongly coupled optical systems, opening up the uncharted terrain of coupling strengths beyond unity by treating the electronic and the photonic components of the system as a single entity. Our theory accounts for the complex interplay of the electronic polarization and the near-field distribution on subwavelength scales, enabling us to exploit the electronic excitation not only to boost the macroscopic polarization, but also to efficiently control the shape of the vacuum mode. Combining a predictive theory with advanced heterostructure growth provides a novel platform for the design of next-generation nanoscale QED systems. With up to $\Omega_{\mathrm{R}} / \omega_{\mathrm{c}}$ $=1.43$, our structures are a key step toward experimental quantum vacuum photonics, enabling the exploration of exotic quantum effects including quantum vacuum phase transitions and quantum vacuum radiation.

\section{ASSOCIATED CONTENT}

\section{S Supporting Information}

The Supporting Information is available free of charge on the ACS Publications website at DOI: 10.1021/acs.nanolett.7b03103.

Description of numerical methods, mode identification procedures, and semiconductor growth (PDF) 


\section{AUTHOR INFORMATION}

\section{Corresponding Author}

*E-mail: christoph.lange@physik.uni-regensburg.de.

\section{ORCID}

Christoph Lange: 0000-0002-2134-6612

\section{Notes}

The authors declare no competing financial interest.

\section{ACKNOWLEDGMENTS}

We thank Simone de Liberato and Cristiano Ciuti for helpful discussions. We gratefully acknowledge support by the European Research Council through grant no. 305003 (QUANTUMsubCYCLE) and the Deutsche Forschungsgemeinschaft (LA 3307/1-1, HU 1598/2-1, BO 3140/3-1, and Collaborative Research Center SFB 689).

\section{REFERENCES}

(1) Thompson, R. J.; Rempe, G.; Kimble, H. J. Phys. Rev. Lett. 1992, $68,1132-1135$

(2) Weisbuch, C.; Nishioka, M.; Ishikawa, A.; Arakawa, Y. Phys. Rev. Lett. 1992, 69, 3314-3317.

(3) Reithmaier, J. P.; Sek, G.; Löffler, A.; Hofmann, C.; Kuhn, S.; Reitzenstein, S.; Keldysh, L. V.; Kulakovskii, V. D.; Reinecke, T. L.; Forchel, A. Nature 2004, 432, 197-200.

(4) Lidzey, D. G.; Bradley, D. D. C.; Skolnick, M. S.; Virgili, T.; Walker, S.; Whittaker, D. M. Nature 1998, 395, 53-55.

(5) Dini, D.; Köhler, R.; Tredicucci, A.; Biasiol, G.; Sorba, L. Phys. Rev. Lett. 2003, 90, 116401.

(6) Günter, G.; Anappara, A. A.; Hees, J.; Sell, A.; Biasiol, G.; Sorba, L.; De Liberato, S.; Ciuti, C.; Tredicucci, A.; Leitenstorfer, A.; Huber, R. Nature 2009, 458, 178-181.

(7) Anappara, A. A.; De Liberato, S.; Tredicucci, A.; Ciuti, C.; Biasiol, G.; Sorba, L.; Beltram, F. Phys. Rev. B: Condens. Matter Mater. Phys. 2009, 79, 201303.

(8) Delteil, A.; Vasanelli, A.; Todorov, Y.; Feuillet Palma, C.; Renaudat St-Jean, M.; Beaudoin, G.; Sagnes, I.; Sirtori, C. Phys. Rev. Lett. 2012, 109, 246808.

(9) Porer, M.; Ménard, J.-M.; Leitenstorfer, A.; Huber, R.; Degl'Innocenti, R.; Zanotto, S.; Biasiol, G.; Sorba, L.; Tredicucci, A. Phys. Rev. B: Condens. Matter Mater. Phys. 2012, 85, 081302.

(10) Wallraff, A.; Schuster, D. I.; Blais, A.; Frunzio, L.; Huang, R.-S.; Majer, J.; Kumar, S.; Girvin, S. M.; Schoelkopf, R. J. Nature 2004, 431, $162-167$.

(11) Niemczyk, T.; Deppe, F.; Huebl, H.; Menzel, E. P.; Hocke, F.; Schwarz, M. J.; Garcia-Ripoll, J. J.; Zueco, D.; Hümmer, T.; Solano, E.; Marx, A.; Gross, R. Nat. Phys. 2010, 6, 772-776.

(12) Yoshihara, F.; Fuse, T.; Ashhab, S.; Kakuyanagi, K.; Saito, S.; Semba, S. Nat. Phys. 2016, 13, 44-47.

(13) Forn-Díaz, P.; García-Ripoll, J. J.; Peropadre, B.; Orgiazzi, J.-L.; Yurtalan, M. A.; Belyansky, R.; Wilson, C. M.; Lupascu, A. Nat. Phys. 2016, 13, 39-43.

(14) Todorov, Y.; Andrews, A. M.; Colombelli, R.; De Liberato, S.; Ciuti, C.; Klang, P.; Strasser, G.; Sirtori, C. Phys. Rev. Lett. 2010, 105, 196402.

(15) Scalari, G.; Maissen, C.; Turcinková, D.; Hagenmüller, D.; De Liberato, S.; Ciuti, C.; Reichl, C.; Schuh, D.; Wegscheider, W.; Beck, M.; Faist, J. Science 2012, 335, 1323-1326.

(16) Maissen, C.; Scalari, G.; Valmorra, F.; Beck, M.; Faist, J.; Cibella, S.; Leoni, R.; Reichl, C.; Charpentier, C.; Wegscheider, W. Phys. Rev. B: Condens. Matter Mater. Phys. 2014, 90, 205309.

(17) Ciuti, C.; Bastard, G.; Carusotto, I. Phys. Rev. B: Condens. Matter Mater. Phys. 2005, 72, 115303.

(18) De Liberato, S. Phys. Rev. Lett. 2014, 112, 016401.

(19) De Liberato, S.; Ciuti, C.; Carusotto, I. Phys. Rev. Lett. 2007, 98, 103602.
(20) Wilson, C. M.; Johansson, G.; Pourkabirian, A.; Simoen, M.; Johansson, J. R.; Duty, T.; Nori, F.; Delsing, P. Nature 2011, 479, 376-379.

(21) Hawking, S. W. Nature 1974, 248, 30-31.

(22) Hagenmüller, D.; Ciuti, C. Phys. Rev. Lett. 2012, 109, 267403.

(23) Zhang, Q.; Lou, M.; Li, X.; Reno, J. L.; Pan, W.; Watson, J. D.; Manfra, M. J.; Kono, J. Nat. Phys. 2016, 12, 1005-1011.

(24) Hepp, K.; Lieb, E. H. Ann. Phys. 1973, 76, 360-404.

(25) Wang, Y. K.; Hioe, F. T. Phys. Rev. A: At., Mol., Opt. Phys. 1973, $7,831-836$

(26) Hagenmüller, D.; De Liberato, S.; Ciuti, C. Phys. Rev. B: Condens. Matter Mater. Phys. 2010, 81, 235303.

(27) Chen, H.-T.; O'Hara, J. F.; Taylor, A. J.; Averitt, R. D.; Highstrete, C.; Lee, M.; Padilla, W. J. Opt. Express 2007, 15, 10841095.

(28) Riek, C.; Seletskiy, D. V.; Moskalenko, A. S.; Schmidt, J. F.; Krauspe, P.; Eckart, S.; Eggert, S.; Burkard, G.; Leitenstorfer, A. Science 2015, 350, 420-423.

(29) Riek, C.; Sulzer, P.; Seeger, M.; Moskalenko, A. S.; Burkard, G.; Seletskiy, D. V.; Leitenstorfer, A. Nature 2017, 541, 376-379.

(30) Eisele, M.; Cocker, T. L.; Huber, M. A.; Plankl, M.; Viti, L.; Ercolani, D.; Sorba, L.; Vitiello, M. S.; Huber, R. Nat. Photonics 2014, $8,841-845$.

(31) Porer, M.; Ménard, J.-M.; Huber, R. Opt. Lett. 2014, 39, 24352438. 